\title{
Current issues in adeno-associated viral vector production
}

\author{
O-W Merten, C Gény-Fiamma and AM Douar \\ Genethon, 1 bis, rue de l'Internationale, Evry, France
}

\begin{abstract}
Adeno-associated virus (AAV) is currently one of the most promising systems for human gene therapy. Numerous preclinical studies have documented the excellent safety profile of these vectors along with their impressive performances in their favored target, consisting of highly differentiated postmitotic tissues such as muscle, central nervous system and liver. Clinical trials have been conducted confirming these data, but also emphasizing the requirement of further high-tech developments of the production and
\end{abstract}

\section{Introduction}

Adeno-associated virus (AAV) based vectors are efficient vehicles for gene therapy in humans. An important set of data has accumulated from various preclinical and clinical models (rodents, dog, monkey), providing evidences of the quasi-absence of pathogenicity or toxicity, an excellent safety profile and the ability to confer long-term gene expression in a wide range of tissues. ${ }^{1}$

\section{Biology of $A A V$}

AAVs are small nonenveloped single-stranded (ss) DNA viruses with a diameter of 18-25 nm. They belong to the Parvoviridae family and are classified in the Dependovirus genus. In total, 11 strains have been isolated and characterised from humans and primates, and new serotypes are continuously discovered. ${ }^{2-6}$ All serotypes share similar structure, genome size and organization, that is, structure and location of the open reading frames (orf), promoters, introns and polyadenylation site. The most divergent serotype is AAV5 with notable differences at the level of the ITR size (167 nucleotides for AAV5 compared to 143-146 for AAV1 to 4 and AAV6) and function. ${ }^{7}$ In addition, at the biological level, they are all dependent on the presence of a helper virus for their replication and gene expression.

AAVs are frequently found in the human populations, $70-80 \%$ of individuals having been exposed to an infectious event.8,9 No known adverse clinical consequences are associated with AAV infection or latency in humans.

The particle is composed of an icosahedric capsid and one single molecule of the viral genome of either polarity, ${ }^{2}$ and has a density of $1.41 \mathrm{~g} / \mathrm{cm}^{3}$ AAVs are very

Correspondence: Dr AM Douar, Genethon, 1 bis, rue de l'Internationale, BP 60, 1002 Evry, France purification procedures that would allow both scaling-up and improvement of vector batch quality, necessary to human application. The scope of this review will be the state of the art in the various production methods of recombinant $A A V$ (rAAV), delimiting their respective perimeter of application and also their main advantages and drawbacks, and thereby shedding light on the main challenges to take in the near future to bring $A A V$ vectors more widely into the clinics. Gene Therapy (2005) 12, S51-S61. doi:10.1038/sj.gt.3302615

resistant to extreme conditions of $\mathrm{pH}$, detergent and temperature, making them easy to manipulate. Finally, wild-type (wt) AAV has the unique property of integration at a specific site into the human genome, which has been extensively described for AAV2. ${ }^{10,11}$ However, because of viral genome manipulation, this propensity is not maintained in vectors.

As the first serotype used to generate vectors, AAV type 2 is so far the best characterised and the majority of gene transfer studies have been based on the use of this serotype. ${ }^{12-14}$ We can and will therefore consider it as a prototype. However, for the last years, rAAV have been actively developed from alternative serotypes. The rational lies in their very similar structure but different tropism and immunological properties, allowing to design a whole set of vectors. The physical characteristics of the different serotypes are close enough to handle them under similar conditions as AAV2 for production and purification. Only the purification systems based on fine capsid structure (electric property, ligands) should be adapted for each serotype. Many groups are actively evaluating the in vivo performances of these serotypes in various animal and disease models (for a recent and extensive review on the serotypes, see Grimm and $\mathrm{Kay}^{15}$ ).

The $4.7 \mathrm{~kb}$ genome of AAV2 (Figure 1) contains two orf, which encode four regulatory proteins, the Rep proteins, and three structural proteins, the Cap proteins. ${ }^{16,17}$ The compacted genome of AAV is framed by two inverted terminal repeats (ITRs), which are basepaired hairpin structures of 145 nucleotides length. The ITRs contain the only necessary regulatory cis acting sequences required by the virus to complete its life cycle, namely the origin of replication of the genome, the terminal resolution site and, the packaging and the integration signals. The two major Rep proteins, Rep78 and Rep68, are involved in viral genome excision, rescue, replication and integration ${ }^{18}$ and also regulate gene 
expression from AAV and heterologous promoters. ${ }^{19,20}$ The minor Rep proteins, Rep52 and Rep40, are involved in replicated ssDNA genome accumulation and packaging. ${ }^{21}$ The cap orf is initiated at the p40 promoter and encodes the three structural capsid proteins VP1, VP2 and VP3. Their stoichiometry in the assembled particle is 1:1:10. Finally, all transcripts share the same polyadenylation signal and equal amounts of virions are found containing strands of plus or minus polarity.
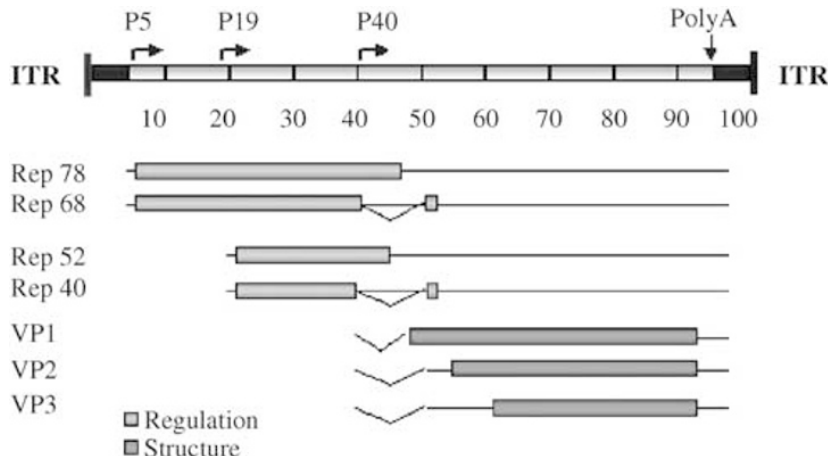

Figure $1 A A V-2$ genome organization. General organization of the genome and genetic elements of AAV type 2 (a scale of 100 map units is used, 1 map unit being equivalent to approximately 47 nucleotides) are shown. The general organization of the other serotypes is similar, T shaped black boxes indicate the ITRs. The horizontal arrows indicate the three transcriptional promoters. The solid lines indicate the transcripts; the introns are shown by the broken lines. A polyadenylation signal present at position 96 is common to all transcripts. The first orf encodes the four regulatory proteins (light grey boxes) arising from the promoters $p 5$ and p19 and the alternative splicing. The second orf (promoter p40) encodes the three capsid proteins (dark grey boxes) from two transcripts. VP-1 is initiated from the first cap transcript, and VP-2 and VP-3 are initiated at two different codon sites from the second cap transcript. Note that the initiation site of VP2 is an ACG.
AAV are naturally replication defective viruses, which made them dependent on the presence of an auxiliary virus in order to achieve their productive cycle. This feature gave the name to the Dependovirus gender. Several viruses can ensure the auxiliary functions: adenovirus (Ad), herpes simplex virus and vaccinia virus $^{22,23}$ as well as cytomegalovirus (CMV). ${ }^{24}$ The infection scheme is fairly simple. Once AAV has entered the cell and been conveyed to the nucleus, its ss genome is converted into a replicative double stranded (ds) form, required for gene expression. In the absence of an auxiliary virus, AAV genome integrates into the host genome and latently persists in a proviral form. In the presence of a helper virus (either by concomitant infection or super infection), the AAV genome can undergo the process of active replication, during which capsid proteins are synthesised and DNA packaged, these steps taking place inside the nucleus. ${ }^{25}$ AAV does not possess a lytic capability by itself, and in the natural infection process, the liberation of AAV virions usually relies on the lytic effect of the helper virus.

\section{$A A V$ vector design}

The design of AAV-based vectors is straightforward, the ITRs being retained, and the exogenous sequences to be transferred cloned in-between. The Rep and Cap functions have therefore to be supplied in trans. Similarly, the helper functions from the auxiliary virus have to be provided (Figure 2 depicts the general principle of rAAV design and production). Several systems coexist, with their own advantages and drawbacks. The next chapters of this review deal with the main methods of rAAV production, that is, the means to provide these functions in a cellular-based production system.

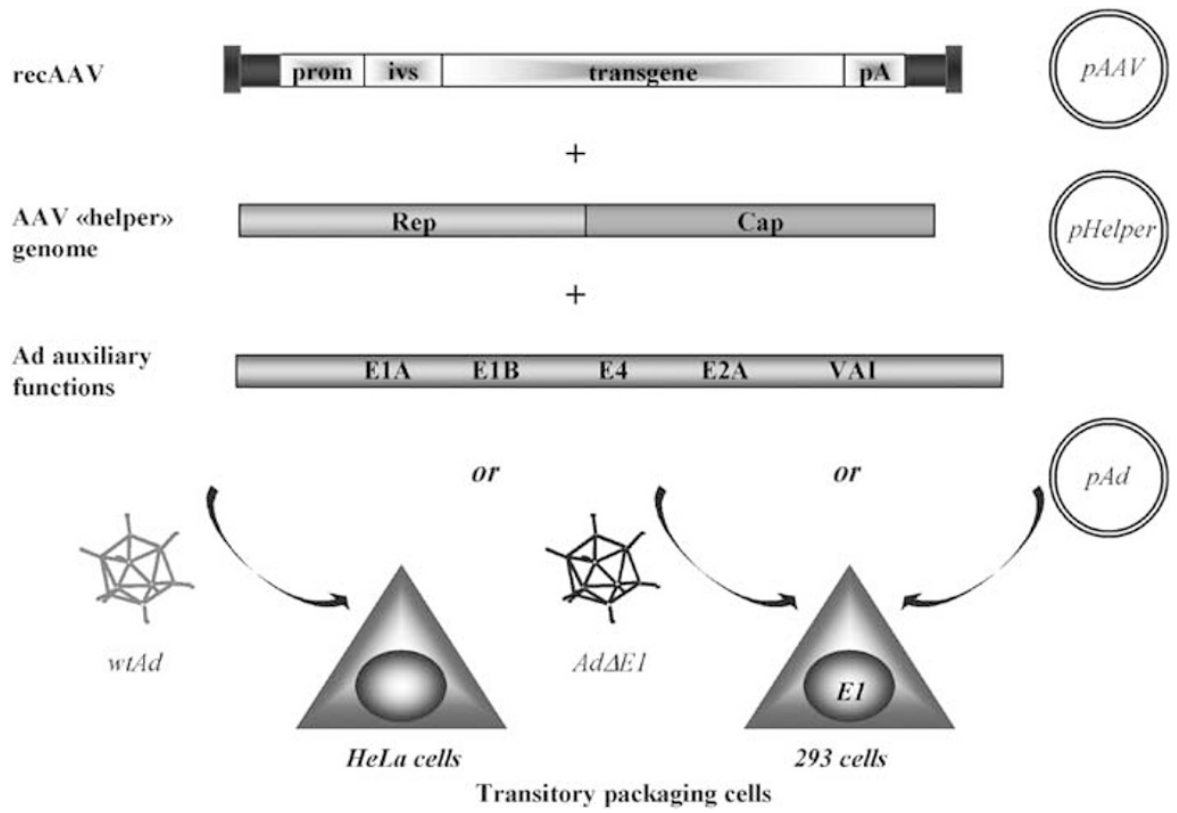

Figure 2 rAAV design and production principle. In a transitory production system, a pAAV Helper and a pAAV plasmids will be brought to the packaging cells by transfection. A second step will consist of the infection of the cells (commonly HeLa cells) with a wtAd or with a first generation E1 deleted Ad in 293 cells (see text for details). An improved Ad free protocol is based on the replacement of Ad infection by the transfection of a mini Ad plasmid along with the $p A A V$ Helper and $p A A V$ plasmids. These methods lead to the generation of about $10^{3}-10^{4}$ particles per cell. Prom: promoter, IVS: intervening sequence (eg intron). 


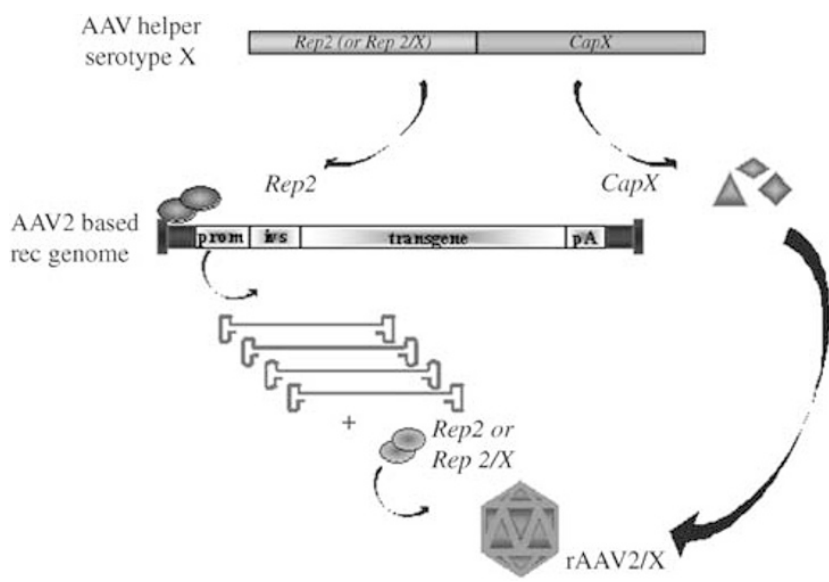

Figure 3 Pseudotyped $r A A V$ design and production principle. Pseudotyped vectors have been extensively and preferentially derived since the early 2000's. The general principle for the genetic trans-complementing system is depicted here. The recombinant AAV genome bearing the expression cassette is framed by serotype 2 ITR. Although the Rep protein from all serotypes but 5 can bind and ensure the replication process, it has been found more convenient and efficient to maintain the rep orf from serotype 2 or to create a chimeric rep gene with its $5^{\prime}$ part of serotype 2 origin and the $3^{\prime}$ of the serotype of interest. ${ }^{82}$ The auxiliary virus functions are provided as for $r A A V 2$ production.

With the discovery and the development of new AAV serotype, the idea has emerged rapidly to develop vectors from these serotypes..$^{3,4,26-28}$ In the vast majority of the studies, a pseudotyping strategy has been adopted for simplicity reason. Basically, all recombinant genomes are based on AAV2 ITRs, and only the capsid is of the serotype of interest (Figure 3). The production strategies for pseudotyped particles are exactly the same as for the classical AAV2 vectors.

In laboratory scale, most of the current methods of production of viral particles are still based on transient transfection of human embryonic kidney (HEK) 293 cells with minor variations. To step toward clinical application, various strategies allowing scaling-up of the amount of virus produced are being actively investigated. Here we shall review and discuss the former and the latter of these approaches.

Although out of the scope of this review, the protocols for purification of rAAV have evolved over the last decade. The most classical and easy to acquire for a nonspecialised laboratory remain the ultracentrifugation on a $\mathrm{CsCl}^{29}$ or iodixanol gradient. ${ }^{30}$ These methods of purification are limited by the capacity of cell lysate volume that can be processed and by the low purity achieved. However, these methods are still widely used and most of the time sufficient for fundamental studies and early in vivo preclinical evaluation of the vectors. More complex techniques based on chromatography are now well developed for the generation of high purity grade and up-scaled production suitable for human clinical application. ${ }^{31-33}$

\section{Traditional methods for the production of $A A V$ vectors}

The classical production methods for $\mathrm{rAAV}$ are based on transient transfection protocols of target/producer cells.
This is a trans-complementation and transitory transfection based approach, which requires the following genetic elements: (i) the sequence of the rAAV genome, usually cloned into a plasmid, called pAAV. It consists of the ITRs framing the expression cassette, which cannot exceed $4.5 \mathrm{~kb}$; (ii) the sequence encoding the two AAV orf rep and cap, usually cloned into a plasmid, called pHelper or pRepCap and complementing the missing rAAV functions and (iii) the required helper functions encoded by the natural auxiliary virus, usually Ad (Figure 2).

\section{Production based on transient transfection and infection}

The first production system of the current AAV vectors, that is, totally deleted for rep and cap orf, was based on a two-plasmids system, pAAV and pHelper, in which overlapping AAV sequences had been totally eliminated, leading to the considerable reduction of wtAAV generation in the recombinant virus batch. ${ }^{34}$ In addition, the exact set of Ad genes providing the auxiliary functions were not clearly identified. This led to the very first method based on double transfection of target cells (HeLa cells) with the pAAV and pHelper, followed by an infection step few hours later with wt Ad at low multiplicity $^{34}$ (Figure 2). After 2-3 days, rAAV accumulation within the cells has reached a maximum and because of the cytolytic effect of Ad, cells are harvested for further purification. It is important in this protocol to harvest the cells before the Ad-induced lysis has occurred, to allow for the purification of rAAV form the cell lysate only. Processing the cellular medium for AAV purification would be extremely laborious due to the limited capacity of ultracentrifuge rotors. This method, in addition of being extremely laborious and time consuming requires extensive purification and heat inactivation, in order to eliminate the contaminating auxiliary wt Ad, a highly undesirable contaminant. Nevertheless, wt Ad contamination of rAAV stocks, associated with the possibility of its propagation, was a persistent problem in the initial studies of gene transfer with $\mathrm{rAAV}$, raising serious questions about the safety of such produced vectors. ${ }^{35}$

Replacement of the wt Ad by a replication defective Ad has been a partial solution to this safety problem, at least limiting the contamination to only nonreplicative Ad. A first generation adenoviral backbone vector deleted for $\mathrm{E} 1$ genes $(\Delta \mathrm{E} 1)$ or thermo-sensitive mutants ${ }^{36,37}$ were used instead of the wt. Because E1 was recognized as being necessary for AAV replication, HEK 293 cells were chosen as the transitory producer cell for AAV vectors. These cells are an Ad5-transformed cell line expressing E1 (E1A and E1B) and are the conventional cells used for recombinant Ad production. In this protocol, 293 cells are transfected with the vector plasmid and the helper plasmid (Figure 2), followed by infection with $\operatorname{Ad} \Delta \mathrm{E} 1$, in similar conditions as those described above based on wt Ad. This method led to reasonably high titers of $10^{11}-10^{12}$ physical particles (also called viral genome, $\mathrm{vg})$ per $\mathrm{ml}\left(10^{2}-10^{3} \mathrm{vg} / \mathrm{cell}\right)$ and also to substantial levels of Ad $\Delta \mathrm{E} 1$ contamination, which is still undesirable because of the toxicity and immunity associated with Ad administration. In addition, heat inactivation does not allow complete Ad elimination and further results in losses in rAAV titers. 
Production based on transient transfection

An important step was achieved in the late 1990s by replacing the $\Delta \mathrm{E} 1$ adenoviral infection step by a mini-Ad plasmid encoding the complementary adenoviral helper functions E2, E4 or $\mathrm{f} 6$ and VAI. ${ }^{37-39}$ This led to the establishment of a tri-transfection method, which allowed for the total elimination of adenoviral particles in the process of production and therefore as a contaminant of AAV preparation (Figure 2). Following a single-step transfection of 293 cells (expressing E1) with the three plasmids at an equimolar ratio, the cells are incubated for $48-72 \mathrm{~h}$ and harvested. However, with such a protocol, a single cell has to be hit three times in order to produce rAAV virions, thereby affecting the efficiency of production on a per-cell basis.

Generating a two-plasmid system made a further improvement of this transfection-only procedure. A two-component packaging plasmid containing the AAV rep and cap genes and the Ad auxiliary functions was generated. ${ }^{40,41}$ This system, requiring only a double-hit cell transfection with the hybrid pAd/Helper and pAAV plasmids, resulted in up to 10 -fold higher titers than those achieved using conventional protocols, production ranging between $10^{2}$ and $10^{4} \mathrm{vg} /$ cell.

\section{Modification of the regulation of rep and cap in the pHelper plasmid}

At the level of the Rep and Cap coding sequences, studies have highlighted the requirement for a tight regulation between rep and cap genes leading to few modifications. It was shown that for optimum virion production per cell, the rep gene must be weakly expressed, whereas the cap gene must be overexpressed, capsid protein being a limiting factor for virion accumulation in the cell. ${ }^{42-44}$ However, it was also shown that capsid protein expression is rate limiting until a threshold of assembled capsids is reached in the cell, and that beyond this limit, efficient encapsidation is more likely to be rate limiting. ${ }^{40}$

With all these transitory methods of production, several important limitations persist. First, they are laborious, and titers within the range of $10^{12-13} \mathrm{vg}$ per $\mathrm{ml}$ are attainable, which translate to about $10^{9-12}$ infectious particles per $\mathrm{ml}$. Such titers, although suitable for most of fundamental biology and preclinical studies in rodent and mid-size animal models, are hardly sufficient for early clinical evaluation in humans, and efficient transfection procedure turned out to be difficult to scale up. Second, 'pseudo-wt AAV' or replication competent (rc) AAV particles arise from nonhomologous recombination between vector and helper AAV plasmids, ${ }^{37,45}$ which is a mater of concern from a safety point of view.

\section{Advanced methods for the production of $A A V$ vectors}

Although the traditional approaches for the production of rAAV are widely used for the production of non-GMP and also GMP grade vectors, they are still affected to their low-scale dimension. The adaptation of transient methods to a reactor environment and the further scaleup to an industrial scale are difficult or almost impossible, although large-scale transient production of recombinant proteins using 293 cells cultivated under serum-free conditions is feasible for developmental purposes. ${ }^{46,47}$ In addition, under GMP-conditions for clinical applications, the transient transfection method requires the certification of several components (plasmid DNA, transfection reagents, and cell lines). Thus, transfection-independent methods have been developed and evaluated for the production of rAAV. These methods are based either on the use of packaging/ producer cell lines, which produce rAAV after induction or on the use of the baculovirus/insect cell system. Finally, another expectation of such methods is to allow for the reduction of $\mathrm{rcAAV}$.

\section{Mammalian packaging and producing systems for rAAV}

Ideally, an AAV packaging system should, in a first instance, circumvent the problem of the transfection process and provide a production technology based on the use of one or several infection steps of the packaging cells containing one or several helper functions. The main approaches for stable mammalian production systems are the packaging cells and producer cells strategies (Figure 4a). The packaging cells approach is an incomplete system, which only harbour a part of all the necessary AAV genetic elements, usually the AAV helper sequences alone (indicated rep-cap in Figure $4 \mathrm{a}$ - packaging cell). The more evolved producer cell lines are complete trans-complementing systems, which bring all necessary AAV derived components, that is, AAV helper sequences (rep-cap) together with the vector sequence (indicated AAV vector in Figure $4 a$ - producer cell). Induction of $\mathrm{rAAV}$ production from a packaging cell line is carried out by either transfection of plasmid containing the rAAV sequence (pAAV) followed by an infection with Ad. As this approach does not alleviate the problem of the scalability associated with transfection, it will not be further developed here. The alternative is to coinfect the packaging cells with a recombinant hybrid $\mathrm{Ad} \Delta \mathrm{E} 1$ bringing in the $\mathrm{rAAV}$ sequence, and wt Ad bringing the early function E1A. When using the producer cells, the production of rAAV only relies on the infection of the cells with a helper virus. If wt Ad or herpesvirus (HSV-1) is to be used to induce the production of rAAV in either of these approaches, the cells concomitantly produce the helper virus.

The choice of the mother cell line to establish the production system depends on several criteria among which its capability to efficiently assemble large quantities of rAAV. In a first glimpse, the 293 seems to be the cell line of choice. However, constitutive expression of Ad E1A gene that activates AAV p5 promoter and expression of the large Rep proteins was shown to be toxic for the cells. ${ }^{48}$ This led to the evaluation of other cell lines, such as HeLa in many studies. In addition, it was suggested that integrated copies of rAAV vectors were more easily rescued from HeLa cells than from 293 cells $^{14}$ and seem to assemble AAV particles more efficiently than 293 cells in a transfection assay. ${ }^{49}$ Other cells such as A549 have also been used to derive packaging and producer cells for rAAV. ${ }^{50,51,63}$

Packaging cell associated with a rAAV/Ad-hybrid virus. In this approach, stable packaging cells containing the AAV rep and cap genes integrated in their genome 
a

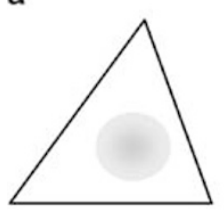

HeLa

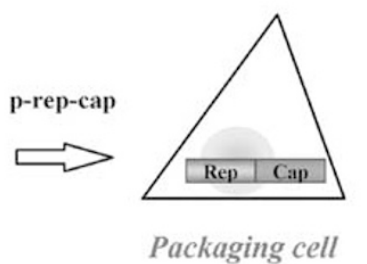

Packaging cell wt Ad5 $\quad$ rAAV/Ad-hybrid
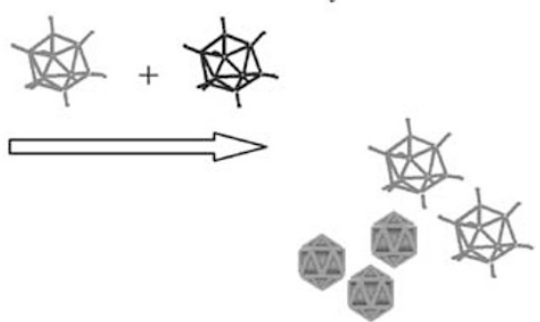

production

pAAV-vector
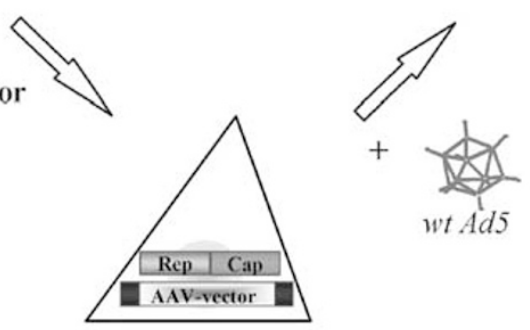

Producer cell

b

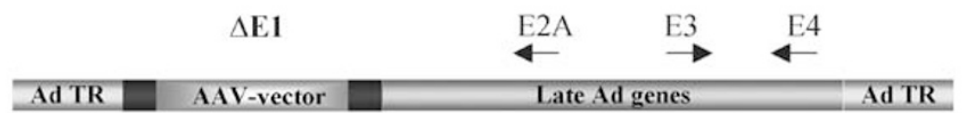

Figure 4 The principle of packaging and producer cell lines. (a) Approaches for HeLa-based packaging and producer cells. The stable transfection of HeLa cells with a plasmid encoding the AAV rep and cap genes leads to a packaging cell line. The transfected HeLa cells can then be used for the production of $r A A V$ by either transfecting them with an pAAVvector (not shown here) followed by the infection with an helper virus (here the Ad) or can be infected twice, first with wt Ad, and later with a rAAV/Ad-hybrid virus. These so infected cells produce in any case wt Ad and rAAV. The stable transfection of the packaging cells with a plasmid containing the $r A A V$ vector or their infection with an $r A A V$ vector leads to a producer cell line. The infection of the producer cell with a helper virus leads to the production of the helper virus, the rescue of the integrated $r A A V$, and its production. (b) Structure of a rAAV/Ad-hybrid vector. A first generation E1 deleted Ad contains the complete AAV vector sequence inserted into the E1 region. AAV ITR are indicated by the black boxes and frame the expression cassette.

are inducibly expressed upon Ad infection. ${ }^{52}$ The yield of rAAV is dependent upon the rep and cap copy number per cell. ${ }^{53,54}$ In these cell lines, the copy number of integrated rep and cap genes, under the control of their native promoters and devoid of ITRs, varies from 5 to 20 per cell. ${ }^{55-57}$ In addition, the rep and cap genes were shown to be rapidly amplified upon infection with wt Ad, up to about 100 copies per cell. ${ }^{58,59}$

In HeLa-based packaging cells, rAAV production is achieved by using a rAAV/Ad-hybrid viral system, in which the rAAV genome is embedded in the replicationdeficient $\triangle \mathrm{E} 1 \mathrm{Ad}$ (Figure 4b). However, this system requires a prior infection with wt Ad to supply the Ad E1 function and activate the native AAV promoters.

The optimal production of rAAV vectors requires the careful optimization of several production parameters, the most important being the MOI for each wt Ad and rAAV / Ad hybrid viruses, the time of infection (TOI) for both viruses, the time interval between both viral infections, and the optimal time to harvest the virus from cell lysate and/or supernatant.

Several studies indicated that a MOI of 10 to 100 for the wt Ad led to optimal rAAV production. ${ }^{55,57}$ Similar range of MOI of the $\mathrm{rAAV} / \mathrm{Ad}$ hybrid virus was shown to be required. Exceeding these MOI led to the reduction of rAAV production, although in various extent, or to the absence of yield improvement, depending on the studies. $^{20,57}$ The differences observed between these studies may be explained by the fact that the packaging cell systems described, although based on HeLa cells, differed in several parameters (different rep and cap constructs inserted).
No specific study has addressed the question of the optimal TOI of the packaging cells. In most protocols, adherent cells were infected $24 \mathrm{~h}$ after plating. ${ }^{55,57}$ However, the rational guide for the choice of the TOI should be the physiological state of the cells, which have to be in their exponential growth phase. One should also pay attention to the time interval between the two viral infections. The cells must be infected first with the wt Ad and 15-24 h later with the $\mathrm{rAAV} / \mathrm{Ad}$ hybrid virus ${ }^{55,57}$ to allow a temporally regulated programme of gene expression, with E1A protein peaking at 20-24 h leading to the induction of high levels of rep and cap expression. Other infection schedules failed to produce high amount of rAAV. ${ }^{57}$

The optimal timing for harvesting rAAV has not been the subject of specific studies in the context of this production system. Generally, rAAV is harvested $72 \mathrm{~h}$ after the initial infection with wt $\mathrm{Ad}^{55}$ then cells are lysed and the cell lysate further processed for downstream purification of rAAV.

The most relevant parameter to give an overall estimate of the system is the final rAAV yield. Under optimised conditions, similar or higher rAAV yields can be obtained with the packaging/rAAV/Ad hybrid system (Table 1) in comparison to the classical transient production methods based on 293 cotransfection or cotransfection/Ad infection. ${ }^{55,57}$

However, the packaging approach offers the main disadvantage of wt helper virus production in parallel to the rAAV. Not only to massively use the cellular resources, thereby no more available for rAAV growth, it leads to the contamination of rAAV stocks, which 
Table 1 Production yields of rAAV using the transient, packaging and producer cells, HSV-1 and baculovirus based systems

\begin{tabular}{|c|c|c|c|c|}
\hline Production method & Yield (vg/cell) & Scale-up & $r C A A V$ & References \\
\hline \multicolumn{5}{|l|}{ Plasmid transfection } \\
\hline 293, transfection+wt Ad5 or Ad $\Delta \mathrm{E} 1$ & $10^{3}-10^{4}$ & & & $\begin{array}{l}34,36,37 \\
55\end{array}$ \\
\hline \multirow[t]{2}{*}{293 , transfection only } & $10-10^{3}$ & & & $37-41$ \\
\hline & & & $<1 \mathrm{rcAAV} / 10^{6} \mathrm{vg}$ & 37 \\
\hline \multicolumn{4}{|l|}{ Packaging cell $+r A A V /$ Ad hybrid } & $52-55,57-59$ \\
\hline HeLa B50+wt Ad5 & $6 \times 10^{3}-2 \times 10^{4}$ & & $<1 \mathrm{rcAAV} / 10^{9} \mathrm{vg}$ & 55 \\
\hline HeLa B50+Ad sub100r & $3 \times 10^{3}-6 \times 10^{5}$ & & & 55 \\
\hline HeLa B50+wtAd5 & & 1001 & & 66 \\
\hline A549 based+wt Ad5 & & & & 50 \\
\hline \multicolumn{4}{|l|}{ Cell line + Ad helper virus } & $58,59,62,63,67,68$ \\
\hline HeLa based+wt Ad5 & $8 \times 10^{3}-5 \times 10^{4}$ & & $<1 \mathrm{rcAAV} / 2 \times 10^{9} \mathrm{vg}$ & 62 \\
\hline HeLa based+wt Ad5 & $10^{3}-2 \times 10^{4}$ & & $<1 \mathrm{rcAAV} / 10^{11} \mathrm{vg}$ & 59 \\
\hline HeLa based+wt Ad5 & $10^{4}-10^{6}$ & & & 49 \\
\hline HeLa based +wt HSV-1 or rHSV & $10^{4}-10^{6}$ & & & 64 \\
\hline A549 based +Ad ts & $>10^{4}$ & 151 & & 50,63 \\
\hline \multicolumn{4}{|l|}{ HSV-1 based system } & 69,70 \\
\hline BHK cells+rHSV & $10^{5}$ & & & 71 \\
\hline \multicolumn{5}{|l|}{ Baculovirus based system } \\
\hline Sf9 cells + rBac & $5 \times 10^{4} 10^{4}-10^{5}$ & 201 & & 80,81 \\
\hline
\end{tabular}

Comparison of different systems and studies is often difficult due to the different methods used to measure the viral particles and the different signification given to units. Infectious units (or particles, i.u. or i.p.) may refer in some studies to transgene expressing cells (such as GFP or LacZ), or to the RCA (recombination centre assay) infectious particles, which measure the capability for a viral particle to enter the cell, traffic up the nucleus, synthesized the complementary strand and being amplified. Infectious particles finally can refer to particles capable of self-production in a cellular system (provided the helper virus is present). Transducing units can be found in many papers, but this measurement is highly dependent on the assay and its sensitivity, as well as the target cells. These methods of measurement are therefore only relevant within identical studies comparing different production systems. As a consequence, viral genome (vg) counting appears to be the most useful approach to compare different studies, as it is based on a physical measure of viral genome against a DNA standard. Here are given the main references for the diverse approaches and when available specific vector production in vg per cell.

therefore requires a highly efficient purification process. To minimise the extent of wt Ad contamination, E1expressing replication deficient Ad were tested with varying results. ${ }^{60}$ Among various temperature-sensitive mutants of E2b gene tested by Gao et al, ${ }^{55}$ some did not affect rAAV yields, or even led to an increased rAAV production (mutant sub100r) in comparison to a wt Ad infection.

Because HeLa is a potentially dangerous cell line due to the presence of the HPV 18 sequence, ${ }^{61}$ other cell lines have been considered to establish packaging cell for rAAV. The human alveolar carcinoma cell line A549based K209, while containing less copies of integrated rep and cap genes than the HeLa-based B50 packaging cell line, also amplified these sequences to similar levels, ${ }^{50}$ depicting an amplification capacity more than 20 times higher than the B50 cells. AAV vector production by staggered infection with a wt Ad and rAAV/Ad-hybrid virus led to comparable productivity. Moreover, the K209 cell line requires optimal wt Ad MOI of 1, significantly lower than for the B50 line, which is an additional advantage in terms of cost and purification requirements.

Tessier et $a l^{51}$ tested two other rep-cap stable cell lines derived from other cell backgrounds (293, TE671). In contrast to HeLa and A549 based packaging cells for which a more than 100- and 2000-fold amplification of rep and cap sequences, respectively, after Ad infection was observed, 293 cells poorly amplified the rep and cap genes (two- to three-fold), whereas no amplification was observed for the TE671 based cells, emphasizing the importance of the cellular background.

As mentioned, the use of the $\mathrm{rAAV} / \mathrm{Ad}$ hybrid virus system in conjunction with packaging cells expressing rep and cap genes offers several benefits over classical transient production systems. In addition to comparable levels in cell-specific production, ${ }^{57}$ it provides a real possibility for a scaling-up to reactor sizes. Another advantage of this production system is its versatility. A single master cell needs to be established and characterised to produce all different AAV vectors, and each desired AAV vector can be easily generated from Ad $\Delta \mathrm{E} 1$, whose generation is a straightforward process. In addition, the formation of rcAAV appears to be reduced with such a system. ${ }^{55,57}$ However, some problems persist, among which the stability of the $\mathrm{rAAV} / \mathrm{Ad}$ hybrid virus, and the question of the acceptability of human-based transformed cell lines (HeLa cells contain papilloma sequences) ${ }^{61}$ for the production of human medicinal products.

rAAV producer cell lines. These approaches are usually based on the above-described packaging cells into which the rAAV sequence has been integrated (Figure 4a). Production of AAV vector solely relies on helper virus infection. Like packaging cells, from which they often derived, producer cells contain the same 1-20 copies of rep and cap gene. ${ }^{50,58,62,63}$ Interestingly, no correlation between rAAV sequence copy number and 
the level of rAAV particles produced was found, with both Ad or HSV-1 as helper. ${ }^{64}$

Establishment of producer cells can be made either by transfection of the packaging cells with a pAAV sequence and a resistance gene (either on the same or a separate plasmid) allowing selection ${ }^{58,59}$ or by infection of the packaging cells with the AAV vector. ${ }^{62,63}$ This latter method requires a rather cumbersome manual selection process of the best clones usually by PCR screening and AAV vector production capacity induced by Ad infection. The overall cloning efficiencies appeared greatly variable between studies and generally low. ${ }^{49,62,63}$

Optimization of producer cells is based on the same parameters as for the packaging cell systems. Wt Ad or replication defective Ad or HSV can be used for the induction of rAAV production. As for the packaging cells, Ad presents the advantage of being moderately pathogenic but the production peaks at about 2-3 days postinfection. Replication-deficient Ads may be used ${ }^{63}$ but were found sometimes instable. ${ }^{49}$ Protease deficient $\mathrm{Ad}(\mathrm{Ad} \Delta \mathrm{PS})^{65}$ offered an alternative but led to reduced levels of rAAV production (our unpublished results). When HSV-1 was used as the helper, it was shown to rapidly induce $\mathrm{rAAV}$ production (which peaks within $24 \mathrm{~h}$ ), leading to similar or higher yields than those obtained with Ad5. ${ }^{64}$ In addition, the action of the HSV-1 is independent of the physiological conditions of the cells (Jenny et al, unpublished results). Finally, screening for various replication defective HSV-1 showed that some of them could induce rAAV assembly at a level similar to that obtained with wt HSV-1 or Ad5. ${ }^{64}$

Optimal TOI of the producer cells with Ad in serumcontaining media is optimum $24-48 \mathrm{~h}$ after the cell plating, the time for the cells to reach the early exponential growth phase. We observed that serum-free conditions rendered the production yields independent of the culture phase and cellular physiology at the TOI when wt Ad or HSV was used (our unpublished results).

As discussed for the packaging cells, Ad infection leads to amplification of rep and cap gene amplification, ${ }^{58}$ as well as the integrated vector genome (more than $10^{6}$-fold) at Ad MOI of 10-100. ${ }^{59}$ Low MOI (1-5) were sufficient to induce rep and cap gene amplification and rAAV assembly with wt HSV-1 or replication-defective HSV-1 strains. ${ }^{64}$

In general, the rAAV production levels reached with these cell lines are within a range of $10^{3}$ to $10^{5} \mathrm{vg}$ per cell upon wt Ad induction ${ }^{56,59,62}$ (for comparison, see Table 1). Similar levels were reached when wt HSV-1 $(\mathrm{MOI}=5)$ was used, with one exceptionally high producer cell line being beyond $10^{6}$ particles per cell. ${ }^{64}$

The single infection step allowed by the producer cell system allows for scaling up to 1301 bioreactors with the HeLa-based B50 cell line ${ }^{66}$ and large-scale production to 151 in serum-free medium in suspension with A549based producer cells. ${ }^{63}$

Other approaches have been taken to establish producer cell lines and should be mentioned here. An inducible HeLa-based cell line containing the AAV rep and cap genes, the AAV vector DNA and the whole Ad5 set of helper functions (E1A-E1B E2, E4, and VA RNA) under the tet-off system was established. However, although rAAV production was achieved, the cell lines were not stable and vector yields were low. ${ }^{67}$
The same authors developed a very elaborated helpervirus-free inducible AAV 293-based producer cell line using conditional disruption of all four rep sequences using the Cre/Lox system. ${ }^{68}$ Upon induction of AAV rep gene expression by an Ad deleted for E1A, E1B and E3 and carrying the cre gene, these producer cells amplified the rep and cap gene five- to ten-fold during vector production. Yields were higher than with the HeLabased cell lines and classical transient transfection methods. In addition, the ratio of $\mathrm{vg} /$ transducing unit was improved compared to other methods. ${ }^{68}$

Producer cell system based on recombinant HSV-1. The minimal set of HSV-1 genes required to provide the auxiliary function to AAV production has been identified as the early genes UL5, UL8, UL52 and UL29. ${ }^{23}$ These genes encode components of the HSV-1 core replication machinery - the helicase, primase and primase accessory proteins and the single-stranded DNA binding protein.

Conway et $a^{69}$ developed a $\mathrm{rAAV}-2$ production system based on the use of a replication defective rHSV-1-(ICP27 deleted) containing AAV rep and cap genes under control of their native promoters (rHSV-1 d27.1-rc). By infecting 293 cells, which had been transiently or stably transfected with a pAAV sequence, high level of all four Rep proteins and rescue of the rAAV from proviral rAAV genome was obtained. High MOI (over 10) were less efficient, likely because of cytotoxicity of immediate early genes. The main advantage of this system is the absence of replication of the rHSV-1 in 293 cells. In addition, this system holds the possibility of a scaleable and flexible production system, conveniently allowing for the production of rAAV with various transgenes (by modifying the producer cell line) or different rAAV serotypes (by modifying the rHSV-1).

A similar approach was developed for the production of rAAV5 vectors, by using a negative rHSV-1- $\Delta \mathrm{ICP} 27$ virus harbouring the AAV5 rep and cap gene. ${ }^{70}$ In contrast to the results obtained for rAAV2 production, ${ }^{69}$ rAAV5 production was significantly lower than by the classic transient transfection method. ${ }^{70}$

Booth et $a^{71}$ recently reported an HSV-1-based rAAV production system in which all functions are supplied by a single virus (bearing the rep-cap genes and the rAAV sequence) or two helper viruses (one with the rep-cap genes and another bearing the rAAV sequence). Nonreplicating (IPC27 deleted) rHSV-1 viruses were compared with replication competent viruses. The most efficient $\mathrm{rAAV}$ production process was that based on the use of nonreplicating HSV / AAV hybrid vectors (Table 1) but required higher MOI. Although these systems offer the advantages of simplicity and scalability, in comparison to the production methods currently used, the presence of HSV-1 derived contaminants will require special attention in the purification process.

The main advantages of producer cell lines lie in the sole need of a single infection step with a helper virus to induce the production of rAAV. Other advantages are that, for industrial GMP production, limited biological components have to be characterised (the cell line and the helper virus), adaptation to serum-free media is possible, large-scale production in bioreactors is achievable, and global yields are at least as good as with the 
classical methods. However, some drawbacks remain. One is the necessity to establish a new producer cell line for each desired AAV vector. In addition, helper virus particles and components contamination of the rAAV stocks is a persistent problem, requiring highly efficient downstream purification methods. Finally, although scaling-up was achieved to some extent for these systems, mammalian cell-based systems may not be the most suitable to reach large-scale production for human application. The baculovirus-insect cell system may thus offer a good alternative.

\section{Production based on the baculovirus expression vector system (BEVS)}

The Baculoviridae are a family of large, enveloped DNA viruses. ${ }^{72}$ Baculoviruses infect preferentially arthropod with the vast majorities of permissive species falling within the order of Lepidoptera. Several continuous cell lines such as Sf9, Sf21 or High Five allowing in vitro baculovirus propagation are commercially available. ${ }^{73}$

Recombinant baculoviruses derived from Autographa californica multinuclear polyhedrosis virus (AcMNPV) are the most commonly used in biotechnology, ${ }^{74}$ in particular for the production of recombinant proteins ${ }^{75}$ or of virus like particles (VLP ie shells devoid of viral nucleic acids). ${ }^{76}$

The main advantages of the BEVS can be summed up as following: (i) the presence of very strong promoters (polyhedrine or p10) enable the production of high quantity of heterologous proteins without gene size limitation; (ii) the insect cells possess the ability to perform the main post-translational modifications, thereby allowing the production of biologically active proteins; and (iii) the baculovirus technology can be easily implemented, scale-up is readily achievable, cells are grown in suspension, and various serum-free media are commercially available.

Assembling viral particles is a more complex process than expressing a single protein. However, it was shown that VLP based on HBV, B19 parvovirus, rotavirus, human papillomavirus could be successfully produced ${ }^{77}$ with the BEVS. It was further demonstrated that during VLP production, the viral structural proteins expressed by baculovirus infected cells could assemble into 3D multimeric structures. ${ }^{76}$

Ruffing et al $^{78}$ described first the possibility to produce AAV-like particles. To study the specific requirements to empty capsid synthesis, the AAV2 capsid proteins were expressed separately on three different recombinant baculovirus, by mutating the internal translational initiation sites of VP2 and/or VP3. As observed for the autonomous parvovirus B19 and canine parvovirus, AAV-based VLP production was achievable with this system, but further pointed to a strict requirement in the minor capsid component VP2 for efficient formation of AAV-like particles in Sf9 cells. In addition, when VLPs were generated from three separate recombinant baculoviruses, the correct VP1:VP2:VP3 stoichiometry of 1:1:10 was not achieved.

The potential of making bioactive recombinant AAV2 using baculovirus as a gene delivery agent has been shown in human 293 cells by Sollerbrant et al. ${ }^{76}$ In this approach, two recombinant baculoviruses harbouring the rep gene and the AAV vector were produced, and the cap gene was inserted into a first generation Ad $\Delta \mathrm{E} 1$. Infection of 293 cells with these two baculoviruses and $\Delta \mathrm{E} 1 \mathrm{Ad}$ led to the production of infectious $\mathrm{rAAV}{ }^{79}$ These results were the first demonstration that an $\mathrm{rAAV}$ sequence cloned into a baculovirus backbone could provide an efficient template for recombinant viral DNA replication and that this replicated $\mathrm{rAAV}$ genome could be efficiently packaged, leading to functional rAAV particles generation. The global yield obtained with this approach was equivalent to that obtained by transient transfection but adherent cells were still used limiting scale up feasibility.

More recently, BEVS has been successfully used for rAAV2 production by Kotin's group. ${ }^{80}$ This strategy consisted of splitting the two trans-complementing functions Rep and Cap and the AAV vector in three different recombinant baculoviruses: rBac-Rep; rBac-Cap and rBac-ITR-GFP-ITR as the rBac-rAAV in this study (Figure 5a). Similar constrains as those mentioned earlier for the classical production system and packaging cell lines stand for the baculovirus system: tight regulation of the Rep proteins is required, by reducing the large Rep78/68 and overexpressing the small Rep52/40. ${ }^{42,44}$ The baculovirus constructions were therefore modified with respect to these constrains.

To establish Rep encoding baculovirus, the large and small rep orf were split and placed under the transcriptional control of two baculovirus promoters of different strength: the polyhedrine promoter from the AcMNPV driving the small Rep52 and the truncated promoter of the immediate-early 1 gene $(\Delta \mathrm{IE} 1)$ of the Orgyia pseudotsugata nuclear polyhedrosis virus driving the large Rep78. Under these conditions, rep52 was highly expressed whereas the expression of Rep78 was reduced, thereby limiting the cytotoxicity of the latter. In addition, the replacement of natural rep promoters by insect promoters allows for the elimination of the helper virus in the production process, thereby improving the overall safety of the system. ${ }^{80}$

The capsid proteins VP1, VP2 and VP3 in the wildtype context are under p40 promoter control and are synthesised from two differently spliced mRNA (Figure 1). In the baculovirus backbone, a native VP expression cassette containing the cap intron failed to produce VP proteins in insect cells. ${ }^{80}$ To circumvent the problem, the p40 promoter was replaced by the AcMNPV polyhedrine promoter. In addition, to maintain the correct VP stoichiometry in Sf9 cells, the ATG codon of VP1 was mutated to ACG and the nine-upstream nucleotide element of the ACG initiation codon of VP2 was introduced at a similar position relative to VP1. These modifications allowed for the expression of all three VP polypeptides from a single transcript without RNA splice and in the correct ratio. ${ }^{80}$

There is no specific modification in the AAV vector sequence cloned into the baculovirus bearing AAV vector: the AAV ITR frame the gene of interest along with the chosen regulatory sequences as in other production systems.

In this system, rAAV2 production is based on a triple infection of the Sf9 cells at their mid-exponential growth phase $\left(\sim 10^{6}\right.$ cells $\left./ \mathrm{ml}\right)$ with each recombinant baculovirus stocks in a $1 / 1 / 1$ stoichiometry with an overall MOI of 5 (Figure 5b). At 3 days postinfection, Sf9 cells and supernatant were harvested and AAV particles were 

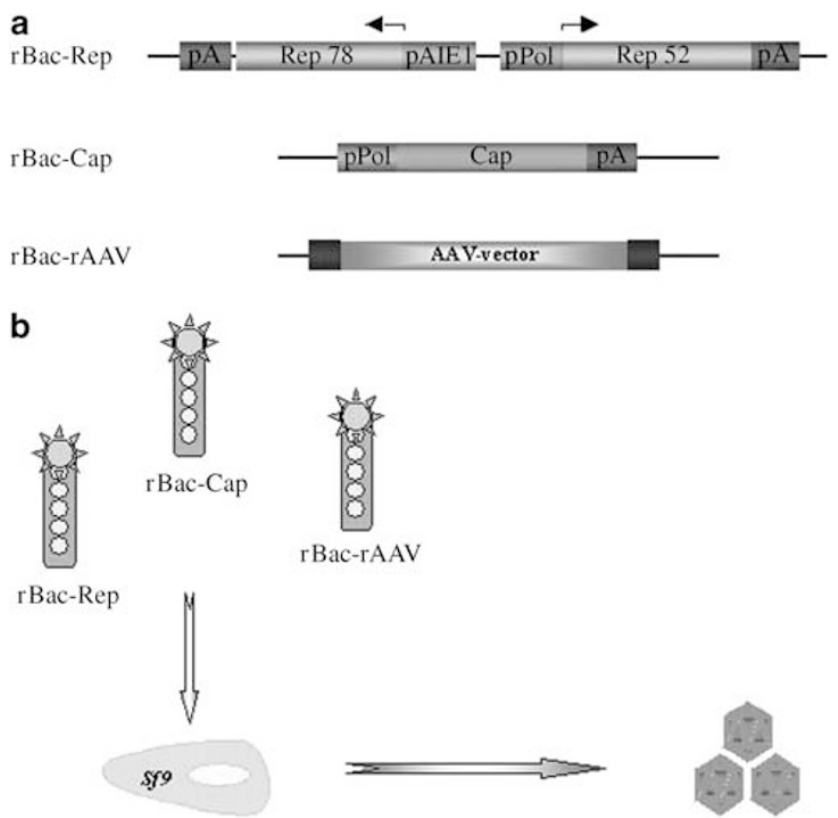

Figure 5 The baculovirus/insect cell production system for $r A A V$. (a) Genetic constructions of the recombinant baculoviruses. The two-split rep orf are driven by two insect promoters, the Polhydrine promoter ( $p$ Pol) of AcMNPV and a truncated form of the immediate-early 1 gene promoter ( $p \Delta E I 1)$ of Orgyia pseudotsugata nuclear polyhedrosis virus. The difference of the promoter strength allows high expression of the small Rep and a reduced expression of the toxic large Rep. rBac-Cap express capsid proteins. The three proteins are directly translated from one transcript. $r B a c-G F P$ carries a $r A A V$-GFP genome. The presence of a $C M V$ and $p 10$ promoter allows GFP expression in both mammalian and insect cell. (b) AAV production is carried out by a triple infection of Sf9 cells. The three recombinant baculoviruses are used at a ratio 1/1/1 with an overall MOI of 5 . At 3 days postinfection $r A A V$ are harvested from cells and supernatant and purified.

purified. Comparison of specific rAAV yield between BEVS and transient transfection showed a nine-fold increase of rAAV production in insect cells $\left(45 \times 10^{3}\right.$ versus $5 \times 10^{3} \mathrm{vg}$ per Sf9 and 293 cells, respectively) (Table 1). Moreover, the SF9-produced rAAV-GFP transduced mouse retinal cells in vivo as efficiently as did 293-produced rAAV-GFP. ${ }^{80}$

We evaluated this system for a pseudotyped rAAV2/1 production. The rBac-Cap encoding AAV1 VP proteins was not initially optimised for the ATG of VP1. AAV2/1 vector was produced as described for rAAV2 (identical cell density, baculoviruses ratio and overall MOI). In this system, the specific production of a rAAV2/1-GFP in Sf9 cells was $10^{4} \mathrm{vg} /$ cell (Geny et al, unpublished data). In vivo evaluation of a rAAV2/1-mSEAP (encoding a secreted form of the murine alkaline phosphatase) produced with the BEVS showed a slight decrease of the mSEAP level (about three-fold) compared to the same vector produced by transient tri-transfection in 293 cells. However, capsid composition analysis with the B1 antibody showed a reduced incorporation of VP1 in AAV capsid. Optimized rBac-Cap at the ATG of VP1 was sufficient to restore a correct ratio of VP proteins and in vivo performances equivalent to those of an rAAV produced by tri-transfection in 293 cells.

The possibility to produce biologically active rAAV with BEVS is the first attractive alternative to conventional methods using transient transfection of adherent mammalian cells. Among the advantages described above, the major points to emphasise are the capacity to produce AAV without scale up limitation, ${ }^{81}$ and the complete absence of the auxiliary virus, thereby considerably improving the safety of the production system. However, this process still presents some drawbacks, among which the genetic instability of the baculovirus stocks, but more importantly the production of baculovirus concomitantly with rAAV. The development of rAAV purification processes will have to be done in parallel to face the increasing production scale and to allow the complete elimination of the recombinant baculoviruses. Finally, common to all production processes, the level of rcAAV that arise with this technology will have to be carefully evaluated.

\section{Conclusions}

Recombinant AAV production has considerably evolved over the last two decades. Although the most promising techniques for a wider application in clinics are in a reachable hand, several issues remain to be solved.

Scaling-up is now an attainable goal, both with producer lines and the baculovirus-based production systems, and similar or higher vector production levels (vg/cell) than with the transfection-based system can be obtained with these advanced production approaches (Table 1). However, the producer cell approaches still hold the question of the stability of the integrated components over the time and the real feasibility of scaling-up in a serum-free, suspension culture system. In addition, theses producer cell lines still require an infectious event with Ad or HSV-1, even if replication defective. This implies the development of highly efficient, large-scale purification methods, which has been largely initiated over the last years, but also to the development of elaborated assays to ensure the absence of auxiliary virus and the establishment of stringent specifications in order to be acceptable by regulatory agencies.

The baculovirus production approach is also very promising, as scaling-up in the absence of serum has been established for the production of other biologicals. However, there are challenges to face: the baculovirus has also to be carefully eliminated in the process of purification and specifications as well as the potential toxicity associated with virus or viral protein contamination has to be carefully evaluated. An additional problem lies in the genetic instability of baculovirus, and it may be necessary to reduce the amount of baculovirus used for the production. For instance, a single baculovirus bearing all genetic elements required for rAAV production may be designed.

Finally, the general problem of rAAV stocks is their contamination with variable amounts of rcAAV, which cannot be eliminated by any purification method. It was shown that these particles arose from nonhomologous recombination between the rAAV and AAV helper (rep and cap) sequences during the process of production. However, these rcAAV could be significantly reduced using a modified helper construct expressing rep and cap genes in opposite direction. ${ }^{45}$ Their presence will have to be carefully measured with these various approaches, and methods allowing their reduction to an acceptable level in term of safety and clinical acceptability will need to be further developed. 


\section{References}

1 Snyder RO. Adeno-associated virus-mediated gene delivery. J Gene Med 1999; 1: 166-175.

2 Berns KI. Parvoviridae: the virus and their replication. In: Fields BN, Knipe DM, Howley PM (eds) Fields in Virology. Lippincott, Raven: Philadelphia, 1996, pp 2173-2197.

3 Rutledge EA, Halbert CL, Russell DW. Infectious clones and vectors derived from adeno-associated virus (AAV) serotypes other than AAV type 2. J Virol 1998; 72: 309-319.

4 Gao GP et al. Novel adeno-associated viruses from rhesus monkeys as vectors for human gene therapy. Proc Natl Acad Sci USA 2002; 99: 11854-11859.

5 Gao $\mathrm{G}$ et al. Clades of Adeno-associated viruses are widely disseminated in human tissues. J Virol 2004; 78: 6381-6388.

6 Mori S, Wang L, Takeuchi T, Kanda T. Two novel adenoassociated viruses from cynomolgus monkey: pseudotyping characterization of capsid protein. Virology 2004; 330: 375-383.

7 Qiu J, Pintel DJ. Alternative polyadenylation of adeno-associated virus type 5 RNA within an internal intron is governed by the distance between the promoter and the intron and is inhibited by U1 small nuclear RNP binding to the intervening donor. J Biol Chem 2004; 279: 14889-14898.

8 Erles K, Sebokova P, Schlehofer JR. Update on the prevalence of serum antibodies (IgG and IgM) to adeno-associated virus (AAV). J Med Virol 1999; 59: 406-411.

9 Tobiasch E et al. Discrimination between different types of human adeno-associated viruses in clinical samples by PCR. J Virol Methods 1998; 71: 17-25.

10 Kotin RM et al. Site-specific integration by adeno-associated virus. Proc Natl Acad Sci USA 1990; 87: 2211-2215.

11 Samulski RJ et al. Targeted integration of adeno-associated virus (AAV) into human chromosome 19. EMBO J 1991; 10: 3941-3950.

12 Samulski RJ, Berns KI, Tan M, Muzyczka N. Cloning of adenoassociated virus into pBR322: rescue of intact virus from the recombinant plasmid in human cells. Proc Natl Acad Sci USA 1982; 79: 2077-2081.

13 Samulski RJ, Chang LS, Shenk T. A recombinant plasmid from which an infectious adeno-associated virus genome can be excised in vitro and its use to study viral replication. J Virol 1987; 61: 3096-3101.

14 Tratschin JD, Miller IL, Smith MG, Carter BJ. Adeno-associated virus vector for high-frequency integration, expression, and rescue of genes in mammalian cells. Mol Cell Biol 1985; 5: 3251-3260.

15 Grimm D, Kay MA. From virus evolution to vector revolution: use of naturally occurring serotypes of adeno-associated virus $(\mathrm{AAV})$ as novel vectors for human gene therapy. Curr Gene Ther 2003; 3: 281-304.

16 Tratschin JD, Miller IL, Carter BJ. Genetic analysis of adenoassociated virus: properties of deletion mutants constructed in vitro and evidence for an adeno-associated virus replication function. J Virol 1984; 51: 611-619.

17 Srivastava A, Lusby EW, Berns KI. Nucleotide sequence and organization of the adeno-associated virus 2 genome. J Virol 1983; 45: 555-564.

18 Weitzman MD, Kyostio SR, Kotin RM, Owens RA. Adenoassociated virus (AAV) Rep proteins mediate complex formation between AAV DNA and its integration site in human DNA. Proc Natl Acad Sci USA 1994; 91: 5808-5812.

19 Horer $\mathrm{M}$ et al. Mutational analysis of adeno-associated virus Rep protein-mediated inhibition of heterologous and homologous promoters. J Virol 1995; 69: 5485-5496.

20 Pereira DJ, McCarty DM, Muzyczka N. The adeno-associated virus $(\mathrm{AAV})$ Rep protein acts as both a repressor and an activator to regulate AAV transcription during a productive infection. I Virol 1997; 71: 1079-1088.
21 King JA, Dubielzig R, Grimm D, Kleinschmidt JA. DNA helicase-mediated packaging of adeno-associated virus type 2 genomes into preformed capsids. EMBO J 2001; 20: 3282-3291.

22 Schlehofer JR, Ehrbar M, zur Hausen H. Vaccinia virus, herpes simplex virus, and carcinogens induce DNA amplification in a human cell line and support replication of a helpervirus dependent parvovirus. Virology 1986; 152: 110-117.

23 Weindler FW, Heilbronn R. A subset of herpes simplex virus replication genes provides helper functions for productive adeno-associated virus replication. J Virol 1991; 65: 2476-2483.

24 McPherson RA, Rosenthal LJ, Rose JA. Human cytomegalovirus completely helps adeno-associated virus replication. Virology 1985; 147: 217-222.

25 Wistuba A et al. Subcellular compartmentalization of adenoassociated virus type 2 assembly. J Virol 1997; 71: 1341-1352.

26 Chiorini JA et al. Cloning of adeno-associated virus type 4 (AAV4) and generation of recombinant AAV4 particles. J Virol 1997; 71: 6823-6833.

27 Chiorini JA, Kim F, Yang L, Kotin RM. Cloning and characterization of adeno-associated virus type 5. J Virol 1999; 73: 1309-1319.

28 Xiao W et al. Gene therapy vectors based on adeno-associated virus type 1. J Virol 1999; 73: 3994-4003.

29 Snyder RO, Xiao X, Samulski RJ. Production of recombinant adeno-associated viral vectors. In: Dracopoli N, Haines J, Krof B, Moir D, Morton C, Seidman C, Seidman J, Smith D (eds). Current Protocols in Human Genetics. John Wiley and Sons Publisher: New York, 1996, pp 11-24.

30 Zolotukhin $\mathrm{S}$ et al. Recombinant adeno-associated virus purification using novel methods improves infectious titer and yield. Gene Therapy 1999; 6: 973-985.

31 Zolotukhin S et al. Production and purification of serotype 1, 2, and 5 recombinant adeno-associated viral vectors. Methods 2002; 28: 158-167.

32 Brument $\mathrm{N}$ et al. A versatile and scalable two-step ion-exchange chromatography process for the purification of recombinant adeno-associated virus serotypes-2 and -5. Mol Ther 2002; 6: 678-686.

33 Kaludov N, Handelman B, Chiorini JA. Scalable purification of adeno-associated virus type 2,4 , or 5 using ion-exchange chromatography. Hum Gene Ther 2002; 13: 1235-1243.

34 Samulski RJ, Chang LS, Shenk T. Helper-free stocks of recombinant adeno-associated viruses: normal integration does not require viral gene expression. J Virol 1989; 63: 3822-3828.

35 Monahan PE et al. Direct intramuscular injection with recombinant AAV vectors results in sustained expression in a dog model of hemophilia. Gene Therapy 1998; 5: 40-49.

36 Snyder RO et al. Persistent and therapeutic concentrations of human factor IX in mice after hepatic gene transfer of recombinant AAV vectors. Nat Genet 1997; 16: 270-276.

37 Salvetti A et al. Factors influencing recombinant adeno-associated virus production. Hum Gene Ther 1998; 9: 695-706.

38 Xiao X, Li J, Samulski RJ. Production of high-titer recombinant adeno-associated virus vectors in the absence of helper adenovirus. J Virol 1998; 72: 2224-2232.

39 Matsushita $\mathrm{T}$ et al. Adeno-associated virus vectors can be efficiently produced without helper virus. Gene Therapy 1998; 5: 938-945.

40 Grimm D et al. Titration of AAV-2 particles via a novel capsid ELISA: packaging of genomes can limit production of recombinant AAV-2. Gene Therapy 1999; 6: 1322-1330.

41 Collaco RF, Cao X, Trempe JP. A helper virus-free packaging system for recombinant adeno-associated virus vectors. Gene 1999; 238: 397-405.

42 Li J, Samulski RJ, Xiao X. Role for highly regulated rep gene expression in adeno-associated virus vector production. J Virol 1997; 71: 5236-5243. 
43 Vincent KA, Piraino ST, Wadsworth SC. Analysis of recombinant adeno-associated virus packaging and requirements for rep and cap gene products. J Virol 1997; 71: 1897-1905.

44 Grimm D, Kern A, Rittner K, Kleinschmidt JA. Novel tools for production and purification of recombinant adenoassociated virus vectors. Hum Gene Ther 1998; 9: 2745-2760.

45 Allen JM, Debelak DJ, Reynolds TC, Miller AD. Identification and elimination of replication-competent adeno-associated virus (AAV) that can arise by nonhomologous recombination during AAV vector production. J Virol 1997; 71: 6816-6822.

46 Schlaeger EJ et al. Transient transfection in mammalian cells. A basic study for an efficient and cost-effective scale up process. In: Merten OW, Perrin P, Griffiths B (eds). New Developments and New Applications in Animal Cell Technology. Kluwer Academic Publishers: Dordrecht, 1998, pp 105-112.

47 Girard P et al. 100-liter transient transfection. Cytotechnology 2002; 38: 15-21.

48 Clark KR, Voulgaropoulou F, Fraley DM, Johnson PR. Cell lines for the production of recombinant adeno-associated virus. Hum Gene Ther 1995; 6: 1329-1341.

49 Blouin $\mathrm{V}$ et al. Improving rAAV production and purification: towards the definition of a scaleable process. J Gene Med 2004; 6 (Suppl 1): S223-S228.

50 Gao GP et al. Rep/Cap gene amplification and high-yield production of AAV in an A549 cell line expressing Rep/Cap. Mol Ther 2002; 5: 644-649.

51 Tessier $\mathrm{J}$ et al. Characterization of adenovirus-induced inverted terminal repeat-independent amplification of integrated adeno-associated virus rep-cap sequences. I Virol 2001; 75: 375-383.

52 Clark KR, Voulgaropoulou F, Johnson PR. A stable cell line carrying adenovirus-inducible rep and cap genes allows for infectivity titration of adeno-associated virus vectors. Gene Therapy 1996; 3: 1124-1132.

53 Inoue N, Russell DW. Packaging cells based on inducible gene amplification for the production of adeno-associated virus vectors. J Virol 1998; 72: 7024-7031.

54 Fan PD, Dong JY. Replication of rep-cap genes is essential for the high-efficiency production of recombinant AAV. Hum Gene Ther 1997; 8: 87-98.

55 Gao GP et al. High-titer adeno-associated viral vectors from a Rep/Cap cell line and hybrid shuttle virus. Hum Gene Ther 1998; 9: 2353-2362.

56 Clark KR, Liu X, McGrath JP, Johnson PR. Highly purified recombinant adeno-associated virus vectors are biologically active and free of detectable helper and wild-type viruses. Hum Gene Ther 1999; 10: 1031-1039.

57 Liu XL, Clark KR, Johnson PR. Production of recombinant adeno-associated virus vectors using a packaging cell line and a hybrid recombinant adenovirus. Gene Therapy 1999; 6: 293-299.

58 Chadeuf $\mathrm{G}$ et al. Efficient recombinant adeno-associated virus production by a stable rep-cap HeLa cell line correlates with adenovirus-induced amplification of the integrated rep-cap genome. J Gene Med 2000; 2: 260-268.

59 Liu X et al. Selective rep-Cap gene amplification as a mechanism for high-titer recombinant AAV production from stable cell lines. Mol Ther 2000; 2: 394-403.

60 Myers MW, Laughlin CA, Jay FT, Carter BJ. Adenovirus helper function for growth of adeno-associated virus: effect of temperature-sensitive mutations in adenovirus early gene region 2. J Virol 1980; 35: 65-75.

61 Schwarz E et al. Structure and transcription of human papillomavirus sequences in cervical carcinoma cells. Nature 1985; 314: 111-114.
62 Mathews LC, Gray JT, Gallagher MR, Snyder RO. Recombinant adeno-associated viral vector production using stable packaging and producer cell lines. Methods Enzymol 2002; 346: 393-413.

63 Farson D et al. Development and characterization of a cell line for large-scale, serum-free production of recombinant adenoassociated viral vectors. J Gene Med 2004; 6: 1369-1381.

64 Toublanc E et al. Identification of a replication-defective herpes simplex virus for recombinant adeno-associated virus type 2 (rAAV2) particle assembly using stable producer cell lines. J Gene Med 2004; 6: 555-564.

65 Oualikene W, Lamoureux L, Weber JM, Massie B. Proteasedeleted adenovirus vectors and complementing cell lines: potential applications of single-round replication mutants for vaccination and gene therapy. Hum Gene Ther 2000; 11: 1341-1353.

66 Thorne B. Scaleable processes for rAAV manufacturing. WilBio Meeting on Viral Vectors \& Vaccines - Process Development and Production Issues, Las Vegas, NE 2003.

67 Qiao $\mathrm{C}$ et al. Feasibility of generating adeno-associated virus packaging cell lines containing inducible adenovirus helper genes. J Virol 2002; 76: 1904-1913.

68 Qiao $C$ et al. A novel gene expression control system and its use in stable, high-titer 293 cell-based adeno-associated virus packaging cell lines. J Virol 2002; 76: 13015-13027.

69 Conway J et al. High-titer recombinant adeno-associated virus production utilizing a recombinant herpes simplex virus type I vector expressing AAV-2 Rep and Cap. Gene Therapy 1999; 6: 986-993.

70 Wustner JT et al. Production of recombinant adeno-associated type 5 (rAAV5) vectors using recombinant herpes simplex viruses containing rep and cap. Mol Ther 2002; 6: 510-518.

71 Booth MJ et al. Transfection-free and scalable recombinant AAV vector production using HSV/AAV hybrids. Gene Therapy 2004; 11: 829-837.

72 Blissard GW. Baculovirus-insect cell interactions. Cytotechnology 1996; 20: 73-93.

73 Schmid G. Insect cell cultivation: growth and kinetics. Cytechnology 1996; 20: 43-55.

74 Kitts PA. Construction of baculovirus recombinants. Cytechnology 1996; 20: 111-123.

75 Ikonomou L, Schneider Y-J, Agathos SN. Insect cell culture for industrial production of recombinant proteins. Appl Microbiol Biotechnol 2003; 62: 1-20.

76 Maranga L, Cruz PE, Aunins JG, Carrondo MJ. Production of core and virus-like particles with baculovirus infected insect cells. Adv Biochem Eng Biotechnol 2002; 74: 183-206.

77 Casal JI. Parvovirus diagnostics and vaccine production in insect cells. Cytotechnology 1996; 20: 261-270.

78 Ruffing M, Zentgraf H, Kleinschmidt JA. Assembly of viruslike particles by recombinant structural proteins of adeno-associated virus type 2 in insect cells. J Virol 1992; 66: 6922-6930.

79 Sollerbrant $\mathrm{K}$ et al. A novel method using baculovirus-mediated gene transfer for production of recombinant adeno-associated virus vectors. J Gen Virol 2001; 82: 2051-2060.

80 Urabe M, Ding C, Kotin RM. Insect cells as a factory to produce adeno-associated virus type 2 vectors. Hum Gene Ther 2002; 13: 1935-1943.

81 Meghrous J et al. Production of recombinant adeno-associated viral vectors using a baculovirus/insect cell suspension culture system: from shake flasks to a 20-L bioreactor. Biotechnol Prog 2005; 21: 154-160.

82 Rabinowitz JE et al. Cross-packaging of a single adenoassociated virus (AAV) type 2 vector genome into multiple AAV serotypes enables transduction with broad specificity. J Virol 2002; 76: 791-801. 